Result Excluding the constant term from the equation, the logarithmic model was the best fit for predicting Kala-azar cases $\left(R^{2}=0.85, p=0.01\right)$. The reported number of Kala-azar cases in 2007 differed from our projected number of cases by 68 . Using a cubic equation it was estimated that there will be 2548 reported Kala-azar cases in Nepal by the year 2015.

Conclusion The number of Kala-azar cases in Nepal is predicted to increase. Curve fitting method could be an effective exploratory modelling technique for predicting cancer frequency and trends over the years. The Kala-azar control programmes should aim to reduce Kala-azar morbidity and prevent mortality by applying a primary healthcare approach which includes active community participation.

\section{P1-323 EVALUATION OF STRATEGIES FOR PANDEMIC INFLUENZA (H1N1) CONTROL AND PREVENTION IN JAPAN BASED ON A TOTAL NUMBER SURVEY IN A CLOSED AREA SIMULATED BY MATHEMATICAL MODELS}

doi:10.1136/jech.2011.142976f.15

\author{
${ }^{1,2} \mathrm{~T}$ Sato, ${ }^{1}{ }^{1} \mathrm{~T}$ Akita, ${ }^{1} \mathrm{~J}$ Tanaka. ${ }^{1}$ Hiroshima University, Graduate School of Biomedical \\ Sciences, Hiroshima, Japan; ${ }^{2}$ Kounu Clinic, Miyoshi, Japan
}

Introduction In 2009, we observed worldwide epidemics of pdmH1N1-flu. We made an almost complete survey of epidemic of pdmH1N1-flu in Kounu town which is isolated from others. All the children with influenza should be reported in Japan for the SchoolHealth-Law preventing their attendance in the infectious period. We applied this epidemic to mathematical models and suggested some strategies to control infection.

Methods Subjects were children of nursery, elementary, and junior high school living in Kounu town. We estimated this epidemic by applying to Kermack-McKendrick model and Multitype epidemic models, selected the most suitable model, and changed the parameters to analyse from three preventing strategies; shortening of virus seeding by 1 day, decreasing of infectivity by $30 \%$, and decreasing the infectivity in especially high infectivity areas to nearly other's one.

Results We estimated $\mathrm{R}_{0}=1.44-6.95$ for the basic reproduction number. The model of assumed subpopulation of school and community was the most suitable, and we found a "super spreader area" on $\mathrm{N}$ area in this model. All the strategies could reduce total infectious population. Only when virus seeding was shortened, epidemic was also shortened. Peak infectious population was much reduced when we intervened in especially high infectivity areas.

Conclusion Most patients were treated with anti-virus and school closure was carried out. This caused the actual epidemic curve shortened and total patients were less than the estimated epidemic model. Therefore, we suggest that particularly where there is a super spreader, to isolate the patients and interruption to infectious roots are effective.

\section{P1-324 CANCER MORTALITY RISK VISUALISATION ON THE AGE- PERIOD SPACE BY REGRESSION MODELS}

\section{doi:10.1136/jech.2011.142976f.16}

${ }^{1} \mathrm{~K}$ Satoh, ${ }^{*}{ }^{2}$ Ken-ichi Kamo, ${ }^{1} \mathrm{~T}$ Tonda. ${ }^{1}$ Research Institute for Radiation Biology and Medicine, Hiroshima University, Hiroshima, Japan; ${ }^{2}$ Center for Medical Education, Sapporo Medical University, Sapporo, Japan

Introduction Cancer information obtained by suitable method becomes a basis of planning the effective cancer control program.
Age-Period-Cohort (APC) model is widely used in the analysis of longitudinal cancer data or prediction. This is constructed by regression model whose explanatory variables are age, period and cohort. However, this model includes the problem that these three elements are not estimated uniquely, because there is a linear dependent relationship among these elements, cf., "cohort = period-age". So there are many kinds of improvements in order to divide these three effects.

Methods To avoid difficulties, we propose to use an interaction model instead of APC model, and visualise cancer risk on the ageperiod space. We compare three models, that is, traditional APC model, geographically weighted regression (GW) model and interaction model. Those models can be described on Poisson regression model. To help understanding those mathematical models, we also show some scripts, which are executable in statistical software, R.

Results and conclusion Applying those regression models to the data of lung cancer in Denmark which is available as lungDK of Epi package in $\mathrm{R}$ and liver cancer in Japan, which is said to have strong cohort effect, cohort effects were visualised in sex effect of Denmark data and in Japanese male data.

\section{P1-325 THE EPIDEMIOLOGY OF COMMUNITY RESILIENCE IN BRIGHTON AND HOVE, ENGLAND}

doi:10.1136/jech.2011.142976f.17

T Scanlon, ${ }^{*} \mathrm{~K}$ Gilchrist. NHS Brighton and Hove, East Sussex, UK

Introduction The current UK spending cuts have brought into stark light the need for the Public Sector to do more with less. A lower cost but more engaged Public Sector is embodied in the political vision of a "Big Society" which seeks to build on individual and community resilience. Knowledge of what community resilience resources are available however, is incomplete.

Methods This study presents the findings from a community resilience mapping exercise undertaken in the spring of 2011, using routine data in Brighton and Hove. The Well-being and Resilience Measure (WARM) Tool was used to map out the epidemiology of community resilience in eight domains:

- Education

- Health

- Material well-being

- Strong and stable families

- Local economy

- Public services

- Crime and antisocial behaviour

- Infrastructure and belonging

Results A series of electoral ward maps with some time trends describes community resilience in these eight domains with reference to four population groups.

- Children and young people

- Working age adults

- Older people

- Natural community groups

Conclusions We believe that this is the first time a Public Sector organisation (NHS Brighton and Hove) in England has comprehensively mapped local resilience using routine data. The approach is timely and the findings are key to understanding the public capacity to deliver the political vision of a 'Big Society' and to implement the 2010 Public Health White Paper "Healthy Lives, Healthy People", which explicitly seeks to "give communities the tools to address their own, particular needs". 